\title{
Nanomaterials-based Biosensors
}

\author{
Arben Merkoçi
}

ICREA \& Institut Català de Nanotecnologia, Nanobioelectronics \& Biosensors Group, Bellaterra, Spain

E-mail: arben.merkoci@icn.cat

\begin{abstract}
:
The need for point of care diagnostic tests has increased enormously in the last few years. Nanomaterials in general and particularly nanoparticles thanks to their optical and electrochemical properties are bringing significant advantages in the design and application of novel biosensors and in this context of diagnostics tests. Biosensors represent an interesting alternative for the development of efficient, fast, low-cost and user-friendly diagnostic devices. Between different biosensing alternatives the nanotechnology and nanomaterial oriented biosensors represent a very attractive tool for clinical applications. Several applications related to DNA, protein and even cell detections based on the use of nonoparticles will be described. The developed devices are based on the use of various platforms which allows their easy applications in diagnostic. The developed devices take advantages of both enhanced electrochemical (i.e. electrocatalytic) or optical (i.e. light absorbance) properties of nanoparticles while being used as labels in immunoreactions or DNA hybridization reactions.
\end{abstract}

Key words: Nanomaterials, biosensors, electrochemical detections, optical detections, DNA, protein cells.

\section{Biosensors and nanomaterials}

The research in biosensor technology is showing a constant increase in relation to the various nanomaterials with interest to be implemented either into transducing or into receptors operation parts so as to enhance the detection sensitivity or improve the multidetection capability beside others.

Metallic nanoparticles (i.e. gold, platinum etc.), quantum dots (i.e. CdS, CdSe etc.), carbon nanotubes, nanowires and recently graphene are in the focus of research of the current nanoscience and nanotechnology. Of special interest between the various nanomaterials are nanoparticles (NPs). NPs with optical or electrochemical properties have led to a variety of optical and electrical detection alternatives such as electrochemical stripping analysis, surface plasmon resonance, inductively coupled plasma mass spectroscopy, fluorescence etc. These NP based alternatives have been applied for protein, DNA or even cell analysis [1-8].

\section{Detection of proteins and cells}

The detection of proteins where immunosensors are playing a key role is of special interest for diagnostics. While enzymes, dyes or even radioactive materials are being used as labels their substitution with more sensitive materials, more robust and safer (in comparison for example to radioactive materials) is of a great concern. Our laboratory has already shown that gold nanoparticles (AuNP) are a very good candidate label for immunosensors. In addition we have demonstrated a direct electrochemical method (differential pulse voltammetry, DPV) that can be used for their direct detection. [1]

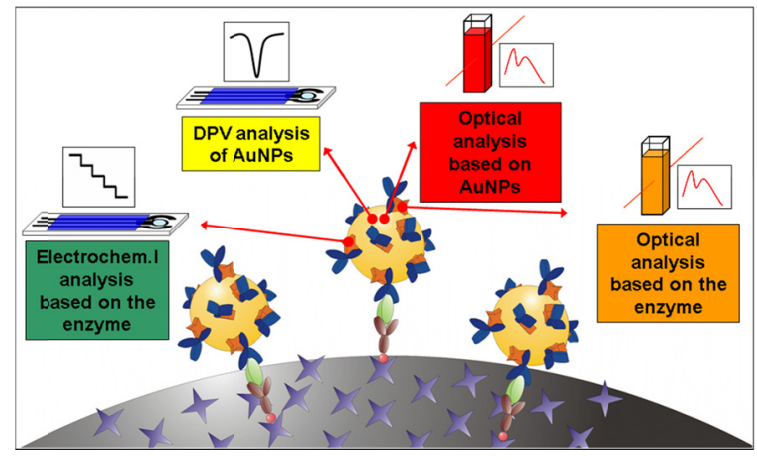

Fig.1. Various electrochemical and optical detection alternatives offered by the use of nanoparticles (gold nanoparticles, AuNP) to detect proteins. AuNP can be detected based on differential pulse voltammetry (DPV), electrochemical analysis based on enzyme activity (i.e. HRP) or optical properties of the same NPs or enzyme. Adapted from reference [1].

The proposed immunoassay is based on the use of magnetic beads modified with streptavidin that are further modified with biotinylated primary antibodies. A sandwich assays is as usually formed after interaction with the protein and a secondary antibody modified with AuNP. The DPV signal of AuNP is 
directly related to the quantity of the 'sandwiched' protein to be detected. In addition given the optical properties of AuNP or of the enzyme (i.e. HRP attached onto its surface) alternative direct \& indirect detection alternatives can also be offered (see Figure 1). AuNPs can also be used as labels in relation to nanochannels platforms. Blocking of antibody modified nanochannels while being incubated with protein containing samples (i.e. blood with a cancer biomarker protein) and a secondary antibody modified with AuNP (further increased with silver) have also been reported as an interesting detection alternative.[9] Figure 2 shows SEM images of the membrane used where red blood cells stand on top of the membrane while the biomarker have penetrated inside the nanochannels and observed through the silver enhanced AuNP used as labels. The system uses an electroactive indicator (Fe 2/3) which penetration is affected by the presence of the protein inside the channel during the immunoreactions.

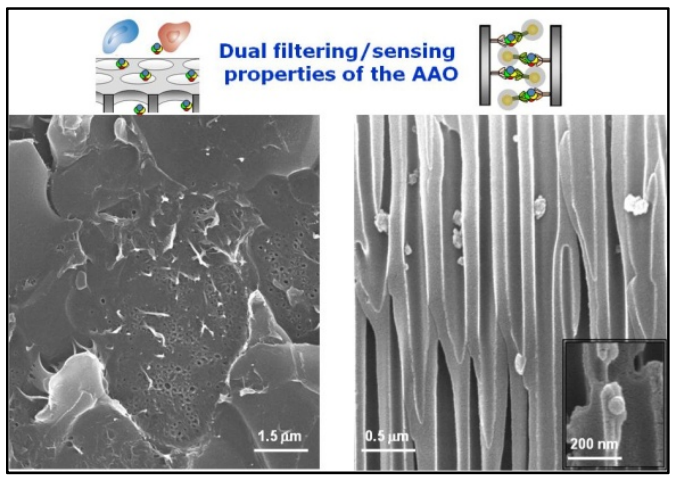

Fig.2. SEM images of the anodized aluminum oxide (AAO) membrane used as both filtering and sensing platform. Adapted from reference [9]

Beside DPV mode based detection the catalytic properties of AuNP in relation to hydrogen evolution reaction are also employed for proteins and even cancer cells detection. [3] Figure 3 shows SEM images of the cancer cells on top of the electrodes used in an AuNP based detection system. The electrocatalytic properties of AuNPs to enhance the hydrogen evolution reaction are explored as an efficient transduction mode of the cell-binding event. This simple system can be used in the detection of surface molecules on tumoral cells and can be further extended to cell suspensions (i.e. circulating cancer cells).

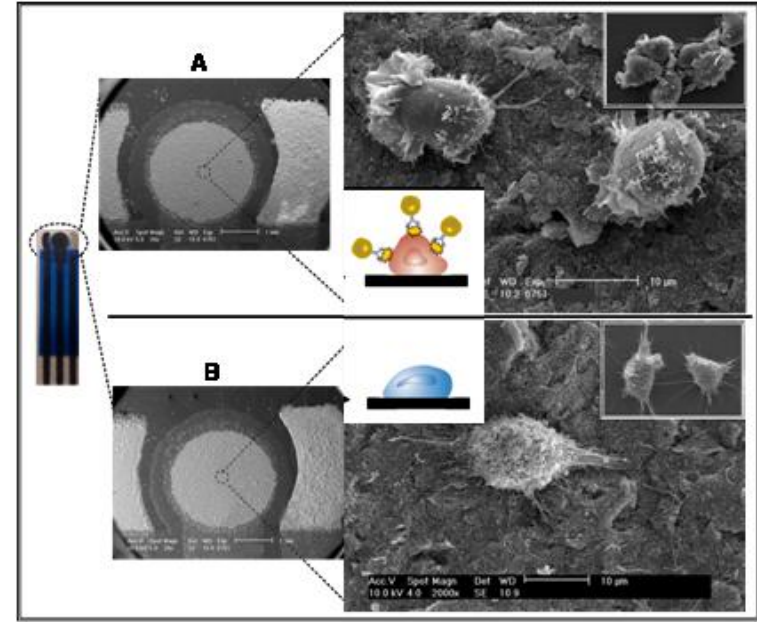

Fig.3. SEM images of the screen-printed carbon electrode (SPCE) transducer (left) with its three surfaces and details of the (A) HMy2 and (B) PC-3 (control) cell lines on the carbon working electrode (right). Inset images correspond to cell growth on the plastic area of the SPCEs. Adapted from reference [3]

\section{Enzymatic biosensors}

Nanoparticles in connection to the use of carbon nanotubes (CNT) can also bring interesting biosensing systems with interest for various applications. Of particular interest is the combination of the electrocatalytic properties of CNT, magnetic properties of nanoparticles (MNPs) and catalytic properties of enzymes. [10] Such a 'synergetic' nano/magneto biocatalytic system can bring additional advantages for uses in lab-on-a-chip systems where an 'on-demand' enzymatic biosystem can be designed so as to achieve an automatic analyzer for multidetection applications. Figure 4 shows a typical response for catechol detection of such a device based on magnetic nanoparticles modified with multi wall carbon nanotubes and tyrosinase (MNP-TyrMWCNT).

Of special interest are nanomaterials for the design of enzyme mimicking biosystems. This is the case of a biosensor developed lastly by our group [11] based on a bimetallic nanowire that 'mimics' enzymes (glucose oxidase) with the added advantage of being able to work even under extreme conditions. The sensor uses a very low electrical potential, thereby minimizing interference and presenting high repeatability and sensitivity. Combining the bimetallic nanowires with a screen-printed transducing platform offers significant advantages in terms of mass production, robustness and stability of the whole biosensing system, making the technology of significant interest for low-cost point-of-care clinical 
analysis and other biotechnological applications.

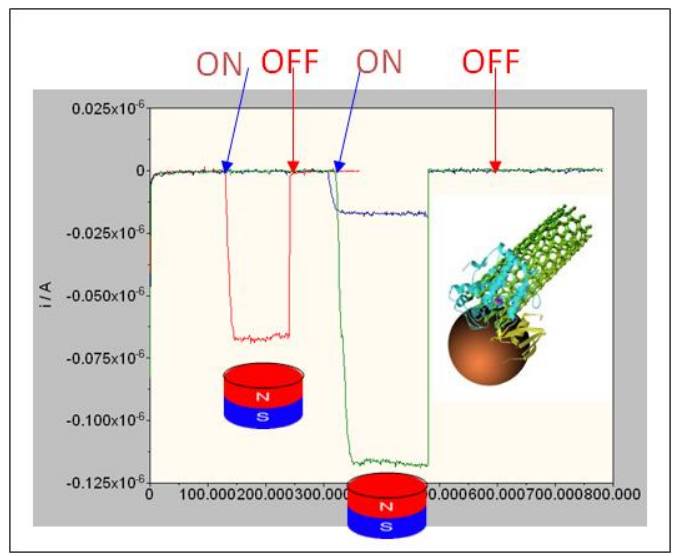

Fig.4. Current-time recordings for catechol obtained for the MNP-Tyr-MWCNT biosensor. Working potential: - $0.15 \mathrm{~V}$; Measurements done in PBS (0.1 $M, p H$ 6.5). Adapted from reference [10].

\section{Conclusions and future perspectives}

Nanomaterials are bringing a series of advantages in the development of novel sensors and biosensors. Between the various kinds of nanomaterials nanoparticles are showing to be very interesting tools in DNA, protein and cell sensing. Their main contribute stands in relation to their use as labels being such a way an advantagous alternative to conventional materials such as enzymes, dyes or radioactive tracers. Probably the most important advantage stand in the variety of detection technologies that can be used thanks to the outstanding optical and electrical properties of nanoparticles beside their stability, multidetection capability and probably cost in comparison for example to enzymes. Their application in optical and electrochemical biosensors is bringing advantages in terms of sensitivity and detection limit (due to various enhancement approaches). This research field is continuously increasing and trying still to solve problems related to real sample applications such as reproducibility, interferences beside aspects related to nanotoxicology overall for in-vivo applications.

\section{Acknowledgements}

We acknowledge MICINN (Madrid) for the projects PIB2010JP-00278 and IT2009-0092, the E.U.'s support under FP7 contract number 246513 "NADINE" and the NATO Science for Peace and Security Programme's support under the project SfP 983807. A.M thanks all his collaborators mentioned in the references of this article.

\section{References}

[1] A. Ambrosi, M.T. Castañeda, A.J, Killard, M.R. Smyth, S. Alegret, A. Merkoçi, Double-Codified Gold Nanolabels for Enhanced Immunoanalysis, Analytical Chemistry 79, 5232-5240 (2007); doi: 10.1021/ac070357m

[2] R. Allabashi, W. Stach, A. De la Escosura-Muñiz, L. Liste-Calleja, A. Merkoçi, ICP-MS - a powerful technique for quantitative determination of gold nanoparticles without previous dissolving, Journal of Nanoparticles Research, 11, 2003-2011 (2009) ; doi: 10.1007/s11051-008-9561-2

[3] A. De la Escosura-Muñiz, C. Sánchez-Espinel, B. Díaz-Freitas, A. González-Fernández, M.M. Da Costa, A. Merkoçi, Rapid identification of tumour cells using a novel electrocatalytic method based in gold nanoparticles, Analytical Chemistry, 81, 10268-10274 (2009) ; doi: 10.1021/ac902087k

[4] A. De la Escosura-Muñiz, M.M. Da Costa, A. Merkoçi, Controlling the electrochemical deposition of silver onto gold nanoparticles: Reducing interferences and increasing the sensitivity of magnetoimmuno assays, Biosensors \& Bioelectronics, 24, 2475-2482 (2009); doi: 10.1016/j.bios.2008.12.028

[5] A. Merkoçi, Nanobiomaterials in Electroanalysis, Editorial, Electroanalysis, 19, 739-741 (2007); doi: 10.1002/elan.200790008

[6] A. Merkoçi, Electrochemical biosensing with nanoparticles, FEBS Journal, 274, 310-316 (2007); doi: 10.1111/j.1742-4658.2006.05603.x

[7] A. Merkoçi, M. Aldavert, S. Marín, S. Alegret, New materials for electrochemical sensing. V. Nanoparticles for DNA labelling, Trends in Analytical Chemistry, 24, 341-349 (2005); doi: 10.1016/j.trac.2004.11.007

[8] A. Merkoçi, M. Aldavert, G. Tarrasón, R. Eritja, S. Alegret, Toward an ICPMS-linked DNA assay based on gold nanoparticles immunoconnected through peptide sequences, Analytical Chemistry, 77, 6500-6503 (2005); doi: 10.1021/ac050539|

[9] A. De la Escosura-Muñiz, A. Merkoçi, A nanochannel / nanoparticle based filtering and sensing platform for direct detection of a cancer biomarker in blood, Small, 7, 675-682 (2011); doi: $10.1002 / s m l l .201002349$

[10] B. Pérez-López, A. Merkoçi, Magnetic Nanoparticles Modified with Carbon Nanotubes for Electrocatalytic Magnetoswitchable Biosensing Applications, Advanced Functional Materials, 21, 255-260 (2011); doi: 10.1002/adfm.201001306

[11] C. Mayorga-Martinez, M. Guix, R.E. Madrid, A. Merkoçi, Chemical Communications, 48, 16861688 (2012); doi: 10.1039/c0xx00000x 